\title{
Einstein, bitte verzeih
}

\section{Werner Niederer}

Dr. med., bis 2003 Chefarzt der Augenabteilung des Spitalzentrums Biel

In der SF1-Sendung «Einstein» vom 6.11.2014 interviewte Tobias Müller den Wissenschaftler Lutz Jäncke, Professor für Neuropsychologie an der Universität Zürich. Weil in diesem Gespräch der gelernte Psychologe auch von Therapie (Psychotherapie) sprach, dachte ich: «Halt, stopp, das geht auch Ärzte etwas an!» Ich schrieb das Interview wortgetreu auf. (Vgl. www.srf. $\mathrm{ch} /$ sendungen/einstein/einstein-spezial/einsteinspezial-grenzen und www.srf.ch/wissen/mensch/ manchmal-ist-mir-sehr-unheimlich-was-da-passiert). Um meinen Kollegen die Lektüre etwas kurzweiliger zu machen, habe ich die im Originalton sehr spärlichen Kommentare des Moderators (TM) etwas erweitert und die Bedeutung seiner Initialen zu Tutanchamun Menes gewandelt. Und nun stellen Sie sich vor, der 90-jährige Ramses II. ( ${ }^{*} 1303, \dagger 1213$ v.Chr.) schicke seinen Kommunikations-Minister TM zu einem Wissenschaftler seines Reiches, der sich rühmt, besser als andere zu wissen, wie der Mensch tickt. Und warum er so tickt. Die Aussagen des altägyptischen Wissenschaftlers sind wortwörtlich jene des Psychologen Lutz Jäncke (LJ). Nichts wurde verändert, nichts hinzugefügt, nichts weggelassen. Die Fragen und Zwischenbemerkungen von Tobias Müller sind kursiv und fett gedruckt, die des erfundenen Ministers Tutanchamun Menes nur kursiv. In seinem schriftlichen Bericht an Ramses hat Menes in eckigen Klammern manchmal ein Wort, von dem er dachte, es könnte dem Pharao nicht geläufig sein, durch Trivialausdrücke ergänzt. Ein guter Minister!

TM: Herr Professor, Sie kennen die rote Karte der Schiedsrichter auf dem Fussballfeld (zeigt dem Professor die rote Karte). Hier ist die rote Karte. Wir wissen nicht, ob die rot ist, geschweige denn, ob Sie das gleiche Rot sehen wie ich? Weshalb denn? [Entschuldige, Ramses, dass ich mein Interview mit einer so blöden und falsch formulierten Frage angefangen habe. Doch das war Absicht. Ich erhoffte mir damit die Belehrung des Professors, Rot sei eine Eigenschaft von Lichtstrahlen, und zwar eine Eigenschaft, die vom menschlichen Auge erkannt werden könne und vom Menschen mit dem Begriff "rot" belegt worden sei; ein Papyrus, der um die Mittagszeit weiss sei, erscheine bei Sonnenuntergang betrachtet rot wie die Sonne; denn er werde rot beleuchtet und könne darum nur rotes Licht zurückwerfen. Werde ein Gegen- stand als rot bezeichnet, hätte der Professor dozieren müssen, so sei die Farbe gemeint, die er hat, wenn die Sonne hoch am Himmel steht. Eine ganz andere Frage sei, welche Emotionen der einzelne Mensch beim Betrachten einer Farbe habe; das hänge davon ab, was für Erinnerungen (bewusst oder unbewusst) eine Farbe in ihm auslöse. Und so weiter. Der Professor aber antwortete:]

LJ: Der Grund dafür ist ganz einfach: Wir interpretieren die Welt. Wir nehmen sie wahr. Und im Wort «Wahr-nehmung» steckt schon drin, dass wir nicht die Realität abbilden, sondern wir interpretieren sie. Und die Interpretation kann höchst individuell sein. Das heisst, die Farbe Rot, die Sie da empfinden, muss nicht gewissermassen die Wahrnehmung von Rot sein, die ich jetzt empfinde.

TM: Was ist der Unterschied von "Interpretieren" und "Abbilden", Herr Professor? Und überhaupt: Was wissen wir denn heute schon über unser Gehirn?

LJ: Also, wir haben enorm viel gelernt. Und die letzten 20 bis 30 Jahre waren eigentlich für die Hirnforschung die Königszeit gewissermassen. Eines der grössten Highlights für mich ist natürlich, dass wir gelernt haben, dass unser Gehirn plastisch ist, sich verändern kann durch Erfahrung.

TM: Also dass wir lernfähig sind. Aber das weiss man doch schon länger als 30 Jahre!

[Ehrlich gesagt, Ramses, hoffte ich, der Professor würden mir etwas Bemerkenswertes erzählen, zum Beispiel, dass Blinde Fähigkeiten entwickeln, die sich Sehende unmöglich aneignen können. Ich denke da, lieber Ramses, an unsere blinde Seherin, die uns stets einige Sekunden zum Voraus sagt, welcher Minister den Thronsaal betreten wird. Ich fragte sie einmal, wie sie das mache, und sie erklärte, sie höre und erkenne die Schritte lange vor uns.]

Entschuldigen Sie, Herr Professor, fahren Sie fort. Das Hirn verändert sich durch Erfahrung.

LJ: In gewisser Weise führt das auch dazu, dass wir zunehmend erkennen müssen und dürfen, dass unser Gehirn ein sehr individuelles Organ ist. Und da bekommt man so ein gewisses Mass von Demut vor der Individualität des Menschen.

TM: Aha! Interessant. Das Hirn ist individuell verschieden. Im Gegensatz zum Herz? In jedem Kopf andere Gedanken? Das könnte dem Pharao natürlich gefährlich werden. Plötzlich könnte es einem Findelkind in den 


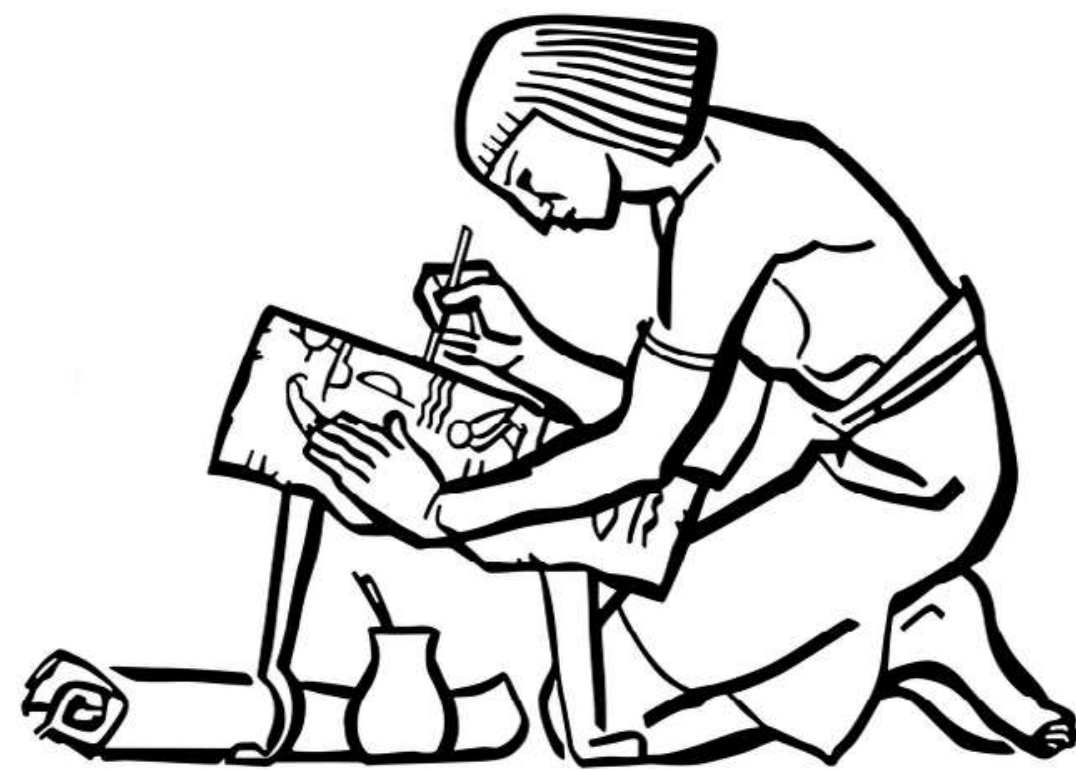

Aufmerksam notiert Kommunikationsminister Tutanchamun Menes (TM) die Antworten des Wissenschaftlers.
TM: Aber eine Möglichkeit, unsere Denkleistung zu erhöhen, wäre natürlich die Kombination von Mensch und Maschine.

LJ: Ja, das ist ja schon Realität. Ich glaube, da sind die wirklichen Freiheitsgrade zu suchen, wo wir als Mensch gewinnen werden.

TM: In der Tat wurden in letzter Zeit viele Werkzeuge erfunden.

LJ: Wir gewinnen durch Kooperation. Wir können als Gruppe viel mehr erreichen. Und das zeigt sich ja auch heute schon: Wir schliessen uns zusammen und teilen unser Wissen und vermehren unser Wissen.

TM: Sie denken da vermutlich an den Kongress in Babylon, der eine markante technische Verbesserung der Metallurgie gebracht hat.

LJ: Und der nächste Punkt ist: Durch die Technik verbessern wir uns in vieler Hinsicht.

TM: Ja, das triff zu: Die neuste Wasseruhrengeneration erlaubt angeblich noch wesentlich genauere Voraussagen der Planetenbewegungen, unterstützt also unsere Hirne ganz wesentlich. Anderseits ist Technik nicht das Wichtigste, was der Mensch braucht, um glücklich zu sein. Was ist denn mit dem Ich hier drin (tippt sich an Kopf und Brust)? Die Seele, das ist doch viel mehr als nur die Summe vieler neuronaler Prozesse. [Neuronale Prozesse $=$ Ereignisse im Hirn.]

LJ: Das hab ich früher auch immer geglaubt. Das ist aber typisch, das wir so denken. Wir kommen aus der Aufklärung [Aufklärung = Erkenntnis, dass wir uns nicht vor dem Teufel, sondern bloss vor teuflischen Menschen zu fürchten brauchen]. Alle haben wir unsere Schulbildung so erhalten. Aber die Aufklärung ist nun mal eben keine Neurowissenschaft, sondern ist eine philosophische Betrachtung des Menschen. TM: Ja, das ist mir schon aufgefallen: Die Neurowissenschaften interessieren sich nicht für die Fähigkeit des Hirns, über das "Sein an sich" nachzudenken, über das Dasein, über die Zeit und Ewigkeit. Sie denken, vermute ich, das bringe ja eh nichts ... Na ja, gut, ich gebe zu, das wäre dann auch schon eine "philosophische Frage", ob Philosophieren etwas «bringt» ...

LJ: Alles das, was wir tun, fühlen, machen, ist das Produkt unseres Gehirns.

TM: Das würde ja bedeuten, in dem Moment, wo ich sterbe, ist nichts mehr.

LJ: Wenn das wirklich so ist, dass unser Bewusstsein das Produkt von neuronalen Prozessen ist ...

TM: «Wenn das wirklich so ist» ... Ja ist es denn nun so? Oder nicht so? - Pardon, ich will Sie nicht unterbrechen. LJ: ... dann ist beim Tode natürlich der neuronale Prozess nicht mehr vorhanden. Und demzufolge haben wir auch nicht so eine Art Seele ... 
TM: Ah so? «Demzufolge»?

LJ: ... eine Art Seele, die irgendwo herumfliegt und woanders gefangen wird.

TM: ... "woanders gefangen"? Interessant. Fühlen Sie sich hier in Ägypten gefangen? Was soll denn gefangen sein? Ihre Sehnsucht nach Freiheit? Die Sehnsucht Ihrer neuronalen Prozesse? (Pause) Na schön, wir haben also keine Seele. Seele ist ein Wort ohne Entsprechung.

Also wäre folglich der Tod die absolute Grenze des Individuums?

LJ: In gewisser Weise schon.

TM: «In gewisser Weise»? Und in welcher Weise nicht? LJ: Aber man darf nicht vergessen, dass ein Mensch auch etwas hinterlässt. Er bleibt im Bewusstsein der Zurückbleibenden erhalten, als Person, die Ideen, die er hinterlassen hat, die Nachkommen, die er hinterlassen hat.

TM: Und wenn die Nachkommen und Ideen auch sterben? Wenn niemand mehr die Erinnerungen pflegt irgendwann wird dies ja der Fall sein -, dann, meinen Sie, ist nichts mehr von all dem, was war? Wer kann dann noch sagen, dass es etwas war, was war? Da wäre ja das Sein an sich nur Illusion. Entschuldigen Sie, ich gleite wieder ab in die nutzlose Philosophie ...

LJ: Insofern ist man Bestandteil eines grossen Ganzen. Und man hat beigetragen zum Entfalten des grossen Ganzen. Und ich glaube, das ist eine sehr positive Botschaft, die eigentlich mit dieser Grenze verbunden ist.

TM: Positiv? Ihre Botschaft lautet doch, das «grosse Ganze», wenn es einst nicht mehr ist, räumt Wall-E auf. [Den Film musst du dir ansehen, lieber Ramses! Gescheit, amüsant, philosophisch.] Und wenn Wall-E samt seinem Bewusstsein zerbricht? Oder meinen Sie, Maschinen würden ewig leben? Wie steht es mit der Intelligenz von Maschinen?

LJ: Ich bin mittlerweile überzeugt, dass Maschinen auch Bewusstsein entwickeln können.

\section{TM: Wieso?}

LJ: Unser Gehirn generiert unser Bewusstsein und das Gehirn ist ein in sich abgeschlossenes physikalisches System ...

TM: Wie? Man kennt doch die Wirkung von Alkohol, Fliegenpilz usw. auf das Denken! So abgeschlossen kann es doch nicht sein. Und vielleicht entdecken die Mediziner in den nächsten viertausend Jahren noch irgendwelche Körpersäfte, die das Verhalten des Menschen beeinflussen. Ja ich möchte sagen: Nicht vielleicht, sicher; denn anders kann man sich zum Beispiel die Wirkung des Kastrierens nicht erklären.

LJ: ... und das Bewusstsein, gewissermassen, das entsteht, ist ja mehr oder weniger ein Epiphänomen, die Folge dieser physikalischen Aktivität ...
TM: Woher wollen Sie das wissen? Es könnte doch auch andersrum sein, dass das Hirn, beziehungsweise seine Aktivitäten ein "Epiphänomen" (welch wunderbare Worthülse!) des Bewusstseins ist, dass das Bewusstsein, wie bei ... - äh, den Namen habe ich vergessen, Hagal, Hagun oder Hegel oder so was - o, pardon, ich gleite schon wieder $a b$ ins Philosophische. Bitte fahren Sie fort.

LJ: ... das heisst, das Gehirn muss in sich gewissermassen arbeiten,...

TM: ... gewissermassen, aha. - Gewissermassen ein Leerlauf?

LJ: ... und das Bewusstsein ist gewissermassen dabei der Geschmack oder Zusatzinformation, aber hat keine Rückwirkung mehr auf die Physik des Gehirns ...

TM: Also das verstehe ich jetzt nicht! Wenn mir «bewusst" wird, dass draussen ein Gewitterregen niedergeht, dann geh ich in die Hütte. Ist das etwa keine Rückwirkung aufs Gehirn?

LJ: ... und wenn Sie das akzeptieren, dann sind Sie gar nicht mehr weit von dem Schritt entfernt, sich zu fragen: Was passiert mit neuronalen Netzwerken, die kleiner sind als das menschliche Gehirn, und da kann man sich also vorstellen, die müssten ja eigentlich auch so etwas wie ein Bewusstsein produzieren ...

TM: Ach soo?! Das Bewusstsein ist ein Saft? Also sind nicht die "neuronalen Prozesse» selbst das Bewusstsein Dieses ist vielmehr ein Produkt, richtig? Ist nicht auch denkbar, dass die "neuronalen Prozesse» das Produkt des Bewusstseins sind ... "Denkbar»? Was sind überhaupt "Gedanken»? Produkt von "neuronalen Prozes sen»? Oder sind "neuronale Prozesse» Produkt von Gedanken? - Pardon! Fahren Sie fort. Wir waren beim Bewusstsein von Maschinen.

LJ: ... da kann man sich also vorstellen, die müssten ja eigentlich auch so etwas wie ein Bewusstsein produzieren, das nicht so ist wie unseres, nicht so reich gefärbt, beispielsweise, auch nicht so differenziert, aber sie müssten eigentlich auch eine Form des Bewusstseins entfalten. Und wenn Sie das akzeptieren, dann bin ich mir ziemlich sicher, wir werden irgendwann mal Gehirne bauen, die durchaus in der Lage sind, so etwas wie ein Bewusstsein zu entfalten.

TM: Da wird's aber auch gefährlich für uns.

LJ: Ja, ich meine, wir müssen einmal darüber nachdenken. In unsern Science-Fiction-Genres [Meditationen über unser künftiges Schicksal] wird immer wieder behauptet, dass die Menschen den Robotermenschen immer überlegen sind. Die Menschen gewinnen immer, und ich glaube, das ist ein grosser Fehler ...

TM: Diesen letzten Satz kann ich mitunterschreiben. Aber nur diesen letzten. 
LJ: ... weil ich vermute, das wird genau andersrum sein, weil wir haben ja heute schon eine Reihe von Computerprogrammen, die uns in allen Dingen überlegen sind.

TM: Oho! Interessant! In ALLEN Dingen? Geographie [Guck mal bei Street View rein, lieber Ramses! Du wirst staunen. Vor Jahrhunderten, zu Menes Zeiten, musste man einen Atlas hervorholen, um zu wissen, wo Amerika liegt, heute sagt es uns die "intelligente Maschine» mit ihrem phänomenalen "Bewusstsein", einem Bewusstsein, das unvergleichlich viel grösser ist als das Bewusstsein eines Atlas] ... ja, ja, die lieben Maschinen sind uns in allen Dingen überlegen, in Musik, Geschichte, Rechnen, Chemie, Physik, Literatur [findet jedes Plagiat in Kürze!], Hilfsbereitschaft [vor allem bei Schulaufgaben] und Emotionen [Emoticons, Smiley und Lätschli], Aggressionen [Malware, Spyware, Trojanerobschon es eigentlich die Griechen waren], Speed-Dating [in Zombiekreisen "Liebe» genannt] und so weiter. Ja, wir müssen gerecht sein und ihnen [den Maschinen] Bewusstsein zugestehen [wäre eigentlich nicht schlecht, "Bewusstsein" einmal zu definieren, oder wenigstens zu versuchen, es zu definieren]. Was immer man einwenden mag: Nur eine Maschine mit Bewusstsein kann uns bis zur Weissglut wütend machen mit Streik, Absturz, schikanöser Fragerei, Rätselinformationen, Indiskretionen, emotionsloser Besserwisserei ... In allen Dingen überlegen, haben Sie gesagt. Bitte fahren Sie fort.

LJ: In den 6oer Jahren sind Computerprogramme entwickelt worden - bereits in den 6oer Jahren! - zur Psychotherapie. Das berühmte Programm «Elisa» ist relativ einfach konstruiert worden und auch sehr erfolgreich eingesetzt worden zur Psychotherapie, und es hat gewissermassen eine bestimmte Form der Psychotherapie modelliert, nämlich die Gesprächspsychotherapie, die nach einem bestimmten Muster abläuft, und wenn Klienten mit diesem Programm geredet hatten, gewissermassen, dann waren sie überwiegend in gleicher positiver Art und Weise gestimmt wie bei einem Gespräch mit einem Therapeuten.

TM: Halt, halt, halt! Das war doch ein erfahrener Psychotherapeut, der «Elisa» beigebracht hat: Wenn der Patient das und das sagt, dann sagst du das und das, und wenn er dies oder jenes sagt, sagst du Satz Nummer $x$, und so weiter. «Von mir aus kannst du auch nur auf Stichworte hören», belehrte der Psychotherapeut Elisa. «Sagt der Pa- tient ‘gestorben` sprichst du sehr mitfühlend den Satz $N r .57$ 'Wir müssen alle sterben, liebe(r) $N$, das ist nun mal so auf dieser Welt. Für alle». Und für 〈N〉 setzt du den Namen ein, den du zu Beginn der Behandlung erfragt hast. Das klingt dann so schön menschlich. Darauffallen sie rein, die Menschen. Natürlich muss der Mensch, der dir seine Stimme leiht, einen weichen, glaubwürdigen, angenehmen Klang haben. Am besten verwendet man die Stimme eines erfahrenen, erfolgreichen Psychotherapeuten, meine Stimme zum Beispiel. Bei gewissen Namen musst du noch nach dem Geschlecht fragen, sonst hast du bei «liebe(r)» ein Problem, was du mit dem eingeklammerten $r$ machen sollst ...». In der Tat, Herr Professor: Die Menschen müssen fürchten, von Ihrer Gilde zu Zombies gestempelt zu werden!

LJ: Und genau das wird auch passieren. Es gibt ja schon die ersten im Internet erhältlichen Therapieprogramme, wo psychisch Kranke sich eben mit einem Computer auseinandersetzen, teilweise im Glauben, es sei ein Psychotherapeut dahinter, dabei ist es ein Computerprogramm ...

TM: O! Auch noch Lügen?! Sie kennen doch den «Vater der Lüge». Nicht? Fahren Sie fort.

LJ: ... und da sehen Sie schon, wie da Intelligenzen sich entfalten. Es gibt da so einen Kollegen, Ray Kurzweil, ein ganz bekannter Wissenschaftler, kein Neurowissenschaftler, eher ein Computerwissenschaftler, der behauptet, dass alsbald die Computerintelligenz, zumindest die gebündelte Computerintelligenz, die menschliche Intelligenz übertreffen wird. Und da sollte man einmal ganz ernsthaft darüber nachdenken. Ich weiss, es fällt auch mir als aufgeklärten Menschen, der noch voll in Descartes'scher, humanistischer Idee ...

TM: [no comment. Kinnlade fällt herunter.]

LJ: ... ausgebildet wurde, schwer zu akzeptieren. Aber es leben bei mir zwei Welten, muss ich wirklich sagen. Ich habe die eine Welt, die noch voll in der Aufklärung [offenbar eine finstere Epoche] drin ist, aber auf der andern Seite habe ich natürlich die neurowissenschaftliche Welt. Und ich sehe, was sich da so alles entfaltet. Und manchmal ist es mir auch sehr unheimlich, was da so passiert. Aber wir müssen da halt einfach mal ernsthaft entgegensteuern.

TM: Gegen Wissenschaft? Gegen die Aufklärung? Na, jaich danke Ihnen für dieses Gespräch. 